\title{
LAGRANGIAN AND HAMILTONIAN FORMULATION OF TRANSMISSION LINE SYSTEMS WITH BOUNDARY ENERGY FLOW
}

\author{
Dimitri Jeltsema \\ Delft Institute of Applied Mathematics \\ Delft University of Technology \\ Mekelweg 4, 2628 CD, Delft, The Netherlands \\ Email: d.jeltsema@tudelft.nl \\ Arjan van der Schaft \\ Institute for Mathematics and Computing Science \\ University of Groningen \\ P.O. Box 800, 9700 AV, Groningen, The Netherlands \\ Email: a.j.van.der.schaft@math.rug.nl \\ (Received \\ 2008)
}

The classical Lagrangian and Hamiltonian formulation of an electrical transmission line is reviewed and extended to allow for varying boundary conditions. The method is based on the definition of an infinite-dimensional analogue of the affine Lagrangian and Hamiltonian input-output systems formulation. The boundary energy flow is then captured in an interaction Lagrangian. This leaves the associated Hamiltonian equations of motion symplectic in form, while the internal Hamiltonian still coincides with the total stored energy in the transmission line. The framework is, however, limited to a line that is terminated on both ends by independent voltage sources. This stems from the fact that the classical formulation captures only one wave equation for a lossless transmission line in terms of an integrated charge density. Additionally, the inclusion of the usual line resistance and shunt conductance via a Rayleigh dissipation function(al) is nontrivial. To circumvent these problems, a family of alternative Lagrangian functionals is proposed. The method is inspired by a (not so well-known) concept from network theory called 'the traditor'.

Keywords: Lagrangian Equations, Hamiltonian Equations, Transmission Line, Boundary Conditions, Distributed-Parameter Systems.

\section{Introduction}

It is well-known that the Lagrangian and Hamiltonian formalism from classical mechanics can be extended to describe a diverse range of lumped- and distributed-parameter 
physical systems. A typical example of such extension is the Lagrangian and Hamiltonian formulation of the wave propagation in an electrical transmission line. Apart from its strong pedagogical value in explaining abstract ideas associated with field theory, transmission lines appear in many applications and are used to interconnect various subsystems that exchange energy among each other. Hence, from an interconnection and control point of view, it is essential to be able to describe a transmission line with varying boundary conditions. However, in extending the classical theory a fundamental difficulty arises in the treatment of boundary conditions. Indeed, the literature seems to be mainly focused on transmission lines with infinite spatial domain, i.e., having infinite length, or having open ports such that the energy exchange through the boundary is zero, e.g., [8] and [12]. The main problem is that for non-zero boundary conditions the spatial differential operator $(\partial / \partial z)$ is not skew-symmetric anymore (since after integration by parts the remaining boundary terms are not zero).

Recently, in the context of Hamiltonian systems, these difficulties are avoided by invoking the notion of an infinite-dimensional Dirac structure [15]. This in turn has led to a class of Hamiltonian boundary control systems - called infinite-dimensional portHamiltonian systems - that generalize the classical (symplectic and Poisson) formulations and allow for non-zero energy flow at the boundary in a mathematically sound way. However, there is no (direct) variational principle (and its resulting Lagrangian equations of motion) involved in this description.

The contributions of this paper can be summarized as follows. First, the classical Lagrangian and Hamiltonian approach is extended by invoking an infinite-dimensional analogue of the affine Lagrangian and Hamiltonian control systems formulation, as originally introduced in [4] (see also [13] for a summary and further developments on the topic). It will turn out that the inclusion of the boundary port variables via so-called interaction Lagrangians and Hamiltonians provides a solution to the boundary energy flow problem. The associated Hamiltonian equations of motion remain symplectic in form, while the internal Hamiltonian still coincides with the total stored energy in the transmission line. Secondly, since the generalized coordinates in the classical Lagrangian formulation of a transmission line are usually associated with the distributed charges, the corresponding equation of motion yields a homogeneous wave equation in terms of a charge wave. As this method is essentially based on the infinite-dimensional analogue of a loop-current analysis, only one of the two transmission line (or telegrapher's) equations (i.e., the voltage balance equation) is described, whereas the other (i.e., the current balance equation) is hidden as a constraint. An additional complication is that the inclusion of the usual line resistance and shunt conductance is far from trivial, if not, impossibleespecially in the nonlinear case. A solution to these problems is presented that invokes the (to our knowledge) not so well-known network-theoretic concept called the traditor, proposed by S. Duinker in the late fifties as part of his development of a complete set of basic network elements [7]. Although we will start the analysis from the foremost simplest version of the traditor, namely the ideal transformer, the concept in itself will lead to a rather novel family of alternative Lagrangian variational principles and associated (symplectic) Hamiltonians. 


\section{Transmission Line Equations}

The fundamental system of partial differential equations that describes the propagation of currents $I(z, t)$ and voltages $V(z, t)$ through a lossless uniform transmission line is given by [11]

$$
\begin{aligned}
& L I_{t}(z, t)+V_{z}(z, t)=0 \\
& C V_{t}(z, t)+I_{z}(z, t)=0,
\end{aligned}
$$

in which $L$ and $C$ represent the distributed inductance and shunt capacitance, respectively. The subscript notation $(\bullet)_{u}$ denotes partial differentiation with respect to some (scalar or vector) variable $u$ (vectors and matrices will be denoted by boldfaced symbols). Both ends of the transmission line are terminated by imposing appropriate boundary conditions at the extremities of the spatial domain $\mathcal{Z}=[0,1]$, where we denote $I^{0}=I(0, t)$, $V^{0}=V(0, t), I^{1}=I(1, t)$, and $V^{1}=V(1, t)$. Differentiating (1) with respect to $z$ and (2) with respect to $t$, we obtain, after some algebraic manipulation, the usual homogeneous wave equation for a lossless uniform transmission line involving either one of the propagation variables $\Phi=\{I, V\}$ :

$$
\Phi_{t t}-\frac{\Phi_{z z}}{L C}=0
$$

When clear from the context, the explicit time- and spatial dependence of the variables will be omitted. Furthermore, for ease of presentation, all variables are assumed to be null at $t \leq 0$.

\subsection{Classical Lagrangian and Hamiltonian Formulation}

As shown in [12], denoting the integrated charge density

$$
Q(z, t)=\int I(z, t) \mathrm{d} t
$$

as the generalized displacement and the current density $Q_{t}(z, t)(=I(z, t))$ as the generalized velocity, the transmission line equations (1)-(2) can be associated with a Lagrangian functional of the form

$$
\mathcal{L}\left(Q, Q_{t}\right)=\int_{\mathcal{Z}} \overline{\mathcal{L}}\left(Q_{t}, Q_{z}\right) \mathrm{d} z,
$$

with Lagrangian density $\overline{\mathcal{L}}\left(Q_{t}, Q_{z}\right)=\frac{1}{2}\left(L Q_{t}^{2}-C^{-1} Q_{z}^{2}\right)$. Indeed, invoking Hamilton's principle

$$
\delta \int_{T} \mathcal{L}\left(Q, Q_{t}\right) \mathrm{d} t=\int_{T} \int_{\mathcal{Z}}\left(-L Q_{t t}+\frac{Q_{z z}}{C}\right) \delta Q \mathrm{~d} z \mathrm{~d} t-\left.\int_{T} \frac{Q_{z}}{C} \delta Q\right|_{z=0} ^{z=1} \mathrm{~d} t=0
$$

with $T=\left[t_{0}, t_{1}\right]$, for $t_{1} \geq t_{0}$, and imposing the boundary condition $-\left.C^{-1} Q_{z}\right|_{\partial \mathcal{Z}}=$ $\left.V\right|_{\partial \mathcal{Z}}=0$, yields a homogeneous wave equation for a lossless transmission line in terms of a charge wave

$$
Q_{t t}-\frac{Q_{z z}}{L C}=0
$$


The Hamiltonian counterpart is obtained by introducing the conjugate momentum $\Pi=\delta_{Q_{t}} \mathcal{L}\left(Q, Q_{t}\right)=L Q_{t}$, where $\delta_{(\bullet)}$ denotes the functional derivative [1, 6], and completing the Legendre transformation $\mathcal{H}(Q, \Pi)$ of $\mathcal{L}\left(Q, Q_{t}\right)$ as

$$
\mathcal{H}(Q, \Pi)=\int_{\mathcal{Z}} \frac{1}{2}\left(\frac{\Pi^{2}}{L}+\frac{Q_{z}^{2}}{C}\right) \mathrm{d} z
$$

Hence, imposing the same boundary condition on $Q_{z}$ as before, the Hamiltonian equations of motion read

$$
\left(\begin{array}{c}
Q_{t} \\
\Pi_{t}
\end{array}\right)=\boldsymbol{F}\left(\begin{array}{c}
\delta_{Q} \mathcal{H}(Q, \Pi) \\
\delta_{\Pi} \mathcal{H}(Q, \Pi)
\end{array}\right)=\left(\begin{array}{c}
\frac{\Pi}{L} \\
\frac{Q_{z z}}{C}
\end{array}\right)
$$

with the symplectic structure matrix

$$
\boldsymbol{F}=\left(\begin{array}{rr}
0 & 1 \\
-1 & 0
\end{array}\right)
$$

Remark 1 Note that the total energy is conserved in the system, i.e., the time-derivative of the total energy vanishes along the trajectories of $(9)$, i.e., $\dot{\mathcal{H}}=0$.

So far, the formalism is in agreement with the standard Lagrangian and Hamiltonian treatment for fields in e.g., [8]. As already pointed out in [12], it is more common to describe wave propagation on a transmission line in terms of currents (or voltages) rather than charges. To bring (7) into correspondence with the conventional wave description (3), first differentiate with respect to time, substitute $Q_{t}=I$, and utilize Clairaut's theorem (i.e., the fact that space and time differentiation can be reversed). Actually, the Euler-Lagrange equation (7) describes just one of the original transmission line equations, namely the voltage balance (1), i.e., $L Q_{t t} \equiv L I_{t}$ and $C^{-1} Q_{z z} \equiv-V_{z}$. Observe that the corresponding current balance (2) follows from the introduction of an integrated flux density

$$
P(z, t)=\int V(z, t) \mathrm{d} t
$$

and the definition of a co-Lagrangian functional, i.e., the dual of (5). We come back to this issue in Section 4.. The main problem, however, is the necessity of the charge to vanish at the boundary which implies a zero boundary energy flow.

Before we generalize the classical approach for the allowance of non-zero boundary energy flow, we first briefly consider the recently proposed infinite-dimensional portHamiltonian formulation.

\subsection{Port-Hamiltonian Approach}

For our transmission line problem, the method in [15] essentially consists in defining both a charge and a flux density, $q(z, t)$ and $\varphi(z, t)$, respectively, so that the total stored 
energy in the transmission line is given as (cf. (8))

$$
\mathcal{H}(q, \varphi)=\int_{\mathcal{Z}} \frac{1}{2}\left(\frac{\varphi^{2}}{L}+\frac{q^{2}}{C}\right) \mathrm{d} z .
$$

The resulting port-Hamiltonian system reads

$$
q_{t}=-\frac{\partial}{\partial z} \delta_{\varphi} \mathcal{H}(q, \varphi)=-\frac{\varphi_{z}}{L} \quad\left(=-I_{z}\right), \quad \varphi_{t}=-\frac{\partial}{\partial z} \delta_{q} \mathcal{H}(q, \varphi)=-\frac{q_{z}}{C} \quad\left(=-V_{z}\right)
$$

together with the boundary variables

$$
\begin{array}{ll}
\left.\delta_{q} \mathcal{H}(q, \varphi)\right|_{z=0}=V^{0}, & \left.\delta_{\varphi} \mathcal{H}(q, \varphi)\right|_{z=0}=I^{0} \\
\left.\delta_{q} \mathcal{H}(q, \varphi)\right|_{z=1}=V^{1}, & \left.\delta_{\varphi} \mathcal{H}(q, \varphi)\right|_{z=1}=I^{1}
\end{array}
$$

Observe that instead of including the spatial differential operator in the Hamiltonian density it is now replacing the symplectic structure, i.e.,

$$
\boldsymbol{F}=\left(\begin{array}{cc}
0 & -\partial / \partial z \\
-\partial / \partial z & 0
\end{array}\right)
$$

Besides its distinct structural simplicity, the port-Hamiltonian formulation does not impose any conditions on the boundary conditions. Hence, the energy flow balance takes the form

$$
\dot{\mathcal{H}}=-\int_{\mathcal{Z}} \frac{\partial}{\partial z}\left(\delta_{\varphi} \mathcal{H}(q, \varphi) \delta_{q} \mathcal{H}(q, \varphi)\right) \mathrm{d} z=-I^{1} V^{1}+I^{0} V^{0},
$$

which is in accordance with Poynting's theorem for a lossless transmission line. Note that for zero boundary energy flow $\dot{\mathcal{H}}=0$. However, as already argued in the Introduction, there is no (direct) variational principle involved in this description.

Remark 2 Note that the charge density $q(z, t)$ differs from the integrated charge density $Q(z, t)$ defined for the Lagrangian (5) in the sense that $q(z, t)=-Q_{z}(z, t)$. This difference can be explained as follows. For the lumped-parameter approximation of the transmission line, as is used in [12] as a starting point (see also Figure 1), the Lagrangian formalism naturally suggests to take the (loop) charge $Q_{k}(t)$ that has passed the $k$-th inductor as the generalized coordinate. This means that the charge associated to the $k$-th capacitor is given as $Q_{k}(t)-Q_{k+1}(t)$, with corresponding voltage $V_{k}(t)=C_{k}^{-1}\left(Q_{k}(t)-Q_{k+1}(t)\right)$. Consequently, in distributed-parameter case, the voltage density $V(z, t)$ corresponds to $-C^{-1} Q_{z}(z, t)$, and thus $q(z, t)=C V(z, t)$.

\section{Classical Formulation Revisited}

In the previous section it is observed that the classical formulation of the transmission line is insufficient in the distinctive case of nonzero boundary energy flow. As will be shown next, this problem can be circumvented introducing an infinite-dimensional analogue of the affine input-output Lagrangian description $[4,13]$. 
Consider again the classical Lagrangian formulation outlined in the previous section and define the triple integrated charge vector

$$
[Q]=\left(\begin{array}{c}
Q \\
Q^{0} \\
Q^{1}
\end{array}\right) .
$$

Denoting

$$
\mathcal{L}^{\text {int }}\left(Q, Q_{t}\right)=\int_{\mathcal{Z}} \underbrace{\frac{1}{2}\left(L Q_{t}^{2}-\frac{Q_{z}^{2}}{C}\right)}_{\overline{\mathcal{L}}^{\mathrm{int}}\left(Q_{t}, Q_{z}\right)} \mathrm{d} z
$$

as the internal Lagrangian, and introducing the interaction Lagrangians $\mathcal{L}^{0}\left(Q^{0}, e^{0}\right)$ and $\mathcal{L}^{1}\left(Q^{1}, e^{1}\right)$, where $e^{0}$ and $e^{1}$ are independent external (control) variables at the boundary. This results in a boundary control Lagrangian functional of the form

$$
\mathcal{L}\left[Q, Q_{t}, e\right]=\mathcal{L}^{\mathrm{int}}\left(Q, Q_{t}\right)+\mathcal{L}^{0}\left(Q^{0}, e^{0}\right)+\mathcal{L}^{1}\left(Q^{1}, e^{1}\right)
$$

Invoking Hamilton's principle, we now obtain

$$
\begin{aligned}
\delta \int_{T} \mathcal{L}\left[Q, Q_{t}, e\right] \mathrm{d} t= & \int_{T} \int_{\mathcal{Z}}\left(-L Q_{t t}+\frac{Q_{z z}}{C}\right) \delta Q \mathrm{~d} z \mathrm{~d} t \\
& +\int_{T}\left\{\left.\left(\mathcal{L}_{Q}^{0}+\frac{Q_{z}}{C}\right) \delta Q\right|_{z=0}+\left.\left(\mathcal{L}_{Q}^{1}-\frac{Q_{z}}{C}\right) \delta Q\right|_{z=1}\right\} \mathrm{d} t=0 .
\end{aligned}
$$

In order to restore to the original equation of motion (7), and since $\left.C^{-1} Q_{z}\right|_{\partial \mathcal{Z}}=-\left.V\right|_{\partial \mathcal{Z}}$, we select $\mathcal{L}^{0}=+Q^{0} E^{0}$ and $\mathcal{L}^{1}=-Q^{1} E^{1}$, where $E^{0}$ and $E^{1}$ denote independent external voltage sources. This establishes a well-posed and mathematically sound variational principle. Moreover, the latter observations directly suggest the definition of a Lagrangian boundary control system of the form

$$
\left(\delta_{\left[Q_{t}\right]} \mathcal{L}\left[Q, Q_{t}, e\right]\right)_{t}-\delta_{[Q]} \mathcal{L}\left[Q, Q_{t}, e\right]=0
$$

where $\delta_{[\bullet]} \mathcal{L}$ is the extended functional derivative [3]

$$
\delta_{[\bullet]} \mathcal{L}=\left(\begin{array}{c}
\overline{\mathcal{L}}_{(\bullet)}-\left(\overline{\mathcal{L}}_{(\bullet)_{z}}\right)_{z} \\
\left.\left(\mathcal{L}_{(\bullet)}^{0}-\overline{\mathcal{L}}_{(\bullet)_{z}}\right)\right|_{z=0} \\
\left.\left(\mathcal{L}_{(\bullet)}^{1}+\overline{\mathcal{L}}_{(\bullet)_{z}}\right)\right|_{z=1}
\end{array}\right)
$$

(Recall that $\overline{\mathcal{L}}$ represents the Lagrangian density.)

Let us next pass on to the Hamiltonian formulation. For the Lagrangian boundary control system (20) one is tempted to define the generalized momentum triple vector

$$
[\Pi]=\delta_{\left[Q_{t}\right]} \mathcal{L}\left[Q, Q_{t}, e\right] .
$$


Since the interaction Lagrangians $\mathcal{L}^{0}$ and $\mathcal{L}^{1}$ are functions of the boundary charges only, the boundary momenta of $[\Pi]$, i.e., $\Pi^{0}$ and $\Pi^{1}$, will vanish identically, which in turn implies that the Lagrangian (18) belongs to the class of so-called singular or degenerate Lagrangians. On the other hand, the internal Lagrangian is non-degenerate since $\delta_{Q_{t}}^{2} \mathcal{L}^{\text {int }}\left(Q, Q_{t}\right)$ is regular. However, by considering the Legendre transformation of the internal and the interaction parts separately, we obtain

$$
\mathcal{H}[Q, \Pi, e]=\mathcal{H}^{\mathrm{int}}(Q, \Pi)+\mathcal{H}^{0}\left(Q^{0}, e^{0}\right)+\mathcal{H}^{1}\left(Q^{1}, e^{1}\right)
$$

where the internal Hamiltonian $\mathcal{H}^{\text {int }}(Q, \Pi)$ is the Legendre transformation of $\mathcal{L}^{\text {int }}\left(Q, Q_{t}\right)$, while the Hamiltonian versions of the interaction terms $\mathcal{H}^{0}\left(Q^{0}, e^{0}\right)=-\mathcal{L}^{0}\left(Q^{0}, e^{0}\right)$ and $\mathcal{H}^{1}\left(Q^{1}, e^{1}\right)=-\mathcal{L}^{1}\left(Q^{1}, e^{1}\right)$. Moreover, differentiating the internal Hamiltonian

$$
\mathcal{H}^{\text {int }}(Q, \Pi)=\int_{\mathcal{Z}} \frac{1}{2}\left(\frac{\Pi^{2}}{L}+\frac{Q_{z}^{2}}{C}\right) \mathrm{d} z,
$$

we now obtain the energy flow balance

$$
\dot{\mathcal{H}}^{\text {int }}(Q, \Pi)=\left.\frac{Q_{z}}{C} Q_{t}\right|_{z=0} ^{z=1}=-I^{1} V^{1}+I^{0} V^{0},
$$

which precisely coincides with (15).

It is important to note that the Hamiltonian equations of motion (9) remain valid since the boundary terms vanish in taking the functional derivatives of $\mathcal{H}[Q, \Pi, e]$ with respect to $Q$ and $\Pi$. This directly relates to the fact that the boundary control Hamiltonian (23) is conserved.

Finally, in a similar fashion as in the case of finite dimensional systems [13], we can define a set of natural outputs for the transmission line system as follows:

$$
\begin{aligned}
y^{0} & =-\mathcal{H}_{e^{0}}[Q, \Pi, e] \\
y^{1} & =-\mathcal{H}_{e^{1}}[Q, \Pi, e] .
\end{aligned}
$$

The selection of $e^{0}=E^{0}$ and $e^{1}=E^{1}$ is tantamount to terminating the transmission line by independent voltage sources (the controls), resulting in $y^{0}=Q^{0}$ and $y^{1}=-Q^{1}$ as the natural outputs. We refer to this particular (causality) configuration as a voltage/voltagecontrolled $(V V)$ transmission line system.

Remark 3 Observe that the dual current/current-controlled $(C C)$ configuration can be obtained starting from an integrated flux coordinate (11) instead of an integrated charge. The transmission line should then be terminated by independent current sources and the natural outputs correspond to the flux-linkages at the terminals.

\section{A Novel Variational Boundary Control Principle}

In the previous section we have accommodated the classical Lagrangian and Hamiltonian formulation to include the practical relevant situation of nonzero boundary energy 
flow. However, this boundary energy flow seems to be restricted to a voltage/voltage controlled configuration due to the form of the natural boundary conditions that come with the formalism. The reason for restriction is directly related with the fact that the Euler-Lagrange equation (7) describes only one of the original transmission line equations, namely the voltage balance (1). Another, but closely related, issue that needs to be solved is the inclusion of the dissipative terms in the case of a lossy transmission line.

In this section, a new family of variational principles is proposed which, in contrast to the classical Lagrangian and Hamiltonian formulation, yields both the transmission line equations (1) and (2) explicitly. In this way, the aforementioned issues can (at least partially) be solved. The inclusion of dissipative terms will be discussed in Section 5.. The construction is strongly inspired by the network-theoretic results presented in [7]. For that reason, we briefly review the main lines of thought in the context of lumpedparameter electrical networks first.

\subsection{Lumped-Parameter Electrical Networks: Loop and Node Lagrangians}

It is well-known, e.g., [5, 10], that for a voltage-driven, possibly nonlinear, lossless LC network consisting of $m$ loops (meshes) the Lagrange equation for each loop takes the form

$$
\frac{\mathrm{d}}{\mathrm{d} t} \mathcal{L}_{\dot{Q}_{k}}^{\text {loop }}-\mathcal{L}_{Q_{k}}^{\text {loop }}=E_{k}, \quad k=1, \ldots, m,
$$

where $E_{k}$ represents a voltage source, $Q_{k}$ represents the loop charge associated to the $k$-th loop, and $I_{k}=\dot{Q}_{k}$ represents the loop current circulating in the $k$-th loop. For this particular case, the Lagrangian function $\mathcal{L}^{\text {loop }}\left(Q_{1}, \ldots, Q_{m}, \dot{Q}_{1}, \ldots, \dot{Q}_{m}\right)$ is given by the difference between the total magnetic co-energy stored in the inductances, $\mathcal{T}^{\text {loop* }}\left(\dot{Q}_{1}, \ldots, \dot{Q}_{m}\right)$, and the total electric energy stored in the capacitors, $\mathcal{U}^{\text {loop }}\left(Q_{1}, \ldots, Q_{m}\right)$, or in short notation: $\mathcal{L}^{\text {loop }}\left(Q_{k}, \dot{Q}_{k}\right)=\mathcal{T}^{\text {loop* }}\left(\dot{Q}_{k}\right)-\mathcal{U}^{\text {loop }}\left(Q_{k}\right)$.

On the other hand, if only current sources are present, then one needs to consider a node (vertex) analysis yielding a so-called co-Lagrange equation associated to each node in the network. Hence, when $J_{j}$ represents a current source and $V_{j}=\dot{P}_{j}$ represents the potential of the $j$-th node together with its time-integral, the flux $P_{j}$, one obtains

$$
\frac{\mathrm{d}}{\mathrm{d} t} \mathcal{L}_{\dot{P}_{j}}^{\text {node* }^{*}}-\mathcal{L}_{P_{j}}^{\text {node* }^{*}}=J_{j}, \quad j=1, \ldots, n
$$

where the co-Lagrangian function $\mathcal{L}^{\text {node* }}\left(P_{1}, \ldots, P_{n}, \dot{P}_{1}, \ldots, \dot{P}_{n}\right)$ is determined by the difference between the total electric co-energy stored in the capacitors, $\mathcal{U}^{\text {node* }}\left(\dot{P}_{1}, \ldots, \dot{P}_{n}\right)$, and the total magnetic energy stored in the inductors, $\mathcal{T}^{\text {node }}\left(P_{1}, \ldots, P_{n}\right)$, or in short notation: $\mathcal{L}^{\text {node* }}\left(P_{j}, \dot{P}_{j}\right)=\mathcal{U}^{\text {node* }}\left(\dot{P}_{j}\right)-\mathcal{T}^{\text {node }}\left(P_{j}\right)$.

\subsection{Mixed-Case: The Traditor}

In the late fifties, Duinker [7] has suggested that in the general case, where both voltage- and current sources may be present, the previous equations can be combined as 
follows

$$
\begin{aligned}
& \frac{\mathrm{d}}{\mathrm{d} t} \mathcal{L}_{\dot{Q}_{k}}-\mathcal{L}_{Q_{k}}=E_{k}, \quad k=1, \ldots, m \\
& \frac{\mathrm{d}}{\mathrm{d} t} \mathcal{L}_{\dot{P}_{j}}-\mathcal{L}_{P_{j}}=J_{j}, \quad j=k+1, \ldots, n,
\end{aligned}
$$

where the network Lagrangian takes the form

$$
\mathcal{L}\left(Q_{k}, P_{j}, \dot{Q}_{k}, \dot{P}_{j}\right)=\underbrace{\mathcal{T}^{\text {loop* }}\left(\dot{Q}_{k}\right)+\mathcal{U}^{\text {node* }}\left(\dot{P}_{j}\right)}_{\text {'kinetic' co-energy }}-\underbrace{\left(\mathcal{U}^{\text {loop }}\left(Q_{k}\right)+\mathcal{T}^{\text {node }}\left(P_{j}\right)\right)}_{\text {'potential' energy }}+\mathcal{S}(\bullet) .
$$

Here, the function $\mathcal{S}$ exclusively contains product terms of the charge, flux, current, and voltage coordinates. Duinker denoted the class of elements that can be associated to this term by the name traditors (derived from the Latin verb tradere, which means to transfer, or to surrender). A general $\eta$-th order traditor is defined as an $\eta$-port element with $\mathcal{S}=f\left(X_{1}, \ldots, X_{\eta}\right) \dot{X}_{\eta}$, in which $f\left(X_{1}, \ldots, X_{\eta}\right)$ is an arbitrary function of appropriate selections from the set of charge and flux coordinates, $Q_{k}$ and $P_{j}$, respectively. A traditor is a non-energic element since it is characterized by the fact that it neither stores nor dissipates energy [2]. This means that at any instant the total power delivered to a traditor is equal to zero, which is also evident from the fact that the associated Hamiltonian, say $\mathcal{S}^{*}$, equals

$$
\mathcal{S}^{*}=\dot{X}_{\eta} \mathcal{S}_{\dot{X}_{\eta}}-\mathcal{S}=0
$$

and thus $\dot{\mathcal{S}}^{*}=0$.

Traditors are defined in various degrees. The simplest examples of a traditor are an open- and short-circuited branch. These two situations are classified as first-degree traditors. Traditors of the second-degree are the ideal transformer and the gyrator. However, in later works, Duinker allocated the name traditor specifically to traditors of the third-degree since these are the simplest to be actually nonlinear and, in addition to the gyrator, synthesize the lower- and higher-degree traditors. The interested reader is referred to [7] for further details. The concept of the traditor will next be used, though in its foremost simplest form, to derive a novel class of variational principles that lead to both the transmission line equations simultaneously. Before we go back to the distributed-parameter transmission line, let us consider the lumped-parameter approximation and the role of the traditor concept in view of its Lagrangian formulation.

Consider the infinitely long chain of linear- and time-invariant LC sections shown in Figure 1. The total kinetic co-energy is found as

$$
\mathcal{T}^{*}\left(\dot{Q}_{k}\right)=\sum_{k} \frac{1}{2} L_{k} \dot{Q}_{k}^{2}
$$

Now, instead of considering the electric energy in terms of the integrated charges, we introduce the electric co-energy in terms of the capacitor voltages $V_{k}=\dot{P}_{k}$, i.e.,

$$
\mathcal{U}^{*}\left(\dot{P}_{k}\right)=\sum_{k} \frac{1}{2} C_{k} \dot{P}_{k}^{2}
$$




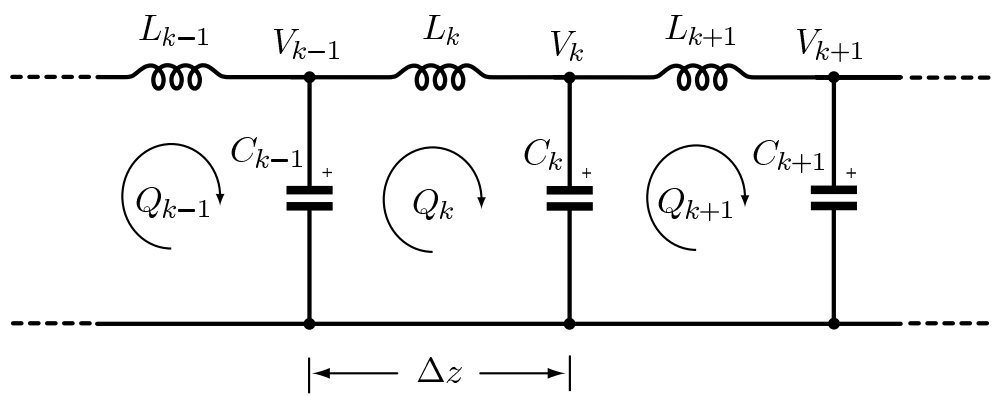

Fig. 1: Infinitely long chain consisting of identical inductors and capacitors $\left(L_{k}=L_{k+1}\right.$, $C_{k}=C_{k+1}$, etc.).

and denote the associated time-integrals $P_{k}$ as the integrated flux coordinates. ${ }^{1}$ With this choice the energy terms $\mathcal{U}\left(Q_{k}\right)$ and $\mathcal{T}\left(P_{k}\right)$ become vacuous, leaving only the Kirchhoff current and voltage laws that define the interconnection between the inductors and the capacitors to be determined.

It turns out that these laws can be captured in the function $\mathcal{S}$ by selecting either

$$
\mathcal{S}(\bullet)=\left\{\begin{array}{l}
\sum_{k} \dot{P}_{k}\left(Q_{k+1}-Q_{k}\right) \\
\sum_{k}^{k} \dot{Q}_{k}\left(P_{k}-P_{k-1}\right) .
\end{array}\right.
$$

Note that the latter function thus reflects the coupling path of the energy transfer between the inductors and the capacitors. For that reason we will denote $\mathcal{S}$ as the coupling Lagrangian. Hence, the network Lagrangian takes the form

$$
\mathcal{L}\left(\bullet, \dot{Q}_{k}, \dot{P}_{k}\right)=\sum_{k} \frac{1}{2} L_{k} \dot{Q}_{k}^{2}+\sum_{k} \frac{1}{2} C_{k} \dot{P}_{k}^{2}+\left\{\begin{array}{l}
\sum_{k} \dot{P}_{k}\left(Q_{k+1}-Q_{k}\right) \\
\sum_{k} \dot{Q}_{k}\left(P_{k}-P_{k-1}\right),
\end{array}\right.
$$

which yields, for either one of the two possible choices for $\mathcal{S}$, the following Lagrangian equations for the $k$-th loop and node

$$
\begin{aligned}
L_{k} \ddot{Q}_{k}+\dot{P}_{k}-\dot{P}_{k-1} & =0 \\
C_{k} \ddot{P}_{k}-\left(\dot{Q}_{k}-\dot{Q}_{k+1}\right) & =0,
\end{aligned}
$$

respectively.

\footnotetext{
${ }^{1}$ Since the number of inductors equals the number of capacitors, we use the same index $k$ for both the inductor and capacitor branches. This means that $Q_{k}, \dot{Q}_{k}$ (resp. $P_{k}, \dot{P}_{k}$ ) are solely associated with the $k$-th inductor (resp. capacitor). The charge (resp. flux) associated to the $k$-th capacitor (resp. inductor) is then determined by $Q_{k}-Q_{k+1}\left(\right.$ resp. $\left.P_{k-1}-P_{k}\right)$.
} 
Remark 4 Note that instead of (34) we could equally take ${ }^{2}$

$$
\mathcal{S}(\bullet)=-\left\{\begin{array}{l}
\sum_{k} Q_{k}\left(\dot{P}_{k}-\dot{P}_{k-1}\right) \\
\sum_{k} P_{k}\left(\dot{Q}_{k+1}-\dot{Q}_{k}\right)
\end{array}\right.
$$

Furthermore, it is also possible to construct a combination of (34) and (38). This results in a set of equivalent 'symmetric' coupling Lagrangians

$$
\mathcal{S}\left(Q_{k}, P_{k}, \dot{Q}_{k}, \dot{P}_{k}\right)=\frac{1}{2}\left\{\begin{array}{l}
\sum_{k} \dot{P}_{k}\left(Q_{k+1}-Q_{k}\right)-\sum_{k} P_{k}\left(\dot{Q}_{k+1}-\dot{Q}_{k}\right) \\
\sum_{k} \dot{Q}_{k}\left(P_{k}-P_{k-1}\right)-\sum_{k} Q_{k}\left(\dot{P}_{k}-\dot{P}_{k-1}\right) .
\end{array}\right.
$$

Interestingly, the latter form coincides with the interconnection terms introduced in the Lagrangian function proposed in [16], where the result is derived in the context of nonlinear lumped electrical networks starting from Brayton-Moser's mixed-potential.

Remark 5 In [17] the lumped-parameter version of a one dimensional transmission line is considered utilizing an implicit port-Lagrangian framework to formulate the equations of motion based on Lagrange-d'Alembert-Pontryagin's principle. Although the resulting equations of motion coincide with the ones presented in (36)-(37), the main differences are (i) that in the present framework the constraints are included in the Lagrangian through the $\mathcal{S}$-function, whereas in the implicit port-Lagrangian framework the constraints are supplemented (thus invoking the need of Lagrange multipliers), and (ii) in contrast to the Lagrangian in [17] the Lagrangian (35) is non-degenerate since

$$
\frac{\partial^{2} \mathcal{L}}{\partial \dot{q}_{i} \partial \dot{q}_{j}}\left(\bullet, \dot{Q}_{k}, \dot{P}_{k}\right)
$$

with $\dot{q}_{i}, \dot{q}_{j} \in\left\{\dot{Q}_{k}, \dot{P}_{k}\right\}$, is non-singular everywhere - allowing us to carry over the results to the Hamiltonian side in a direct manner.

\subsection{Alternative Lagrangian Functionals}

In a similar fashion as in [12], we proceed from a lumped- to a distributed-parameter transmission line formulation by considering the limiting case in which the spacing $\Delta z$ between the LC sections becomes infinitesimal. For zero boundary energy flow this means that (35) becomes a functional

$$
\mathcal{L}\left(\bullet, Q_{t}, P_{t}\right)=\int_{\mathcal{Z}} \frac{1}{2}\left(L Q_{t}^{2}+C P_{t}^{2}\right) \mathrm{d} z+\mathcal{S}(\bullet)
$$

\footnotetext{
${ }^{2}$ Of course, in the case of infinitely many LC sections both the total sums in (34) and (38) coincide. However, in view of their respective distributed-parameter versions we aim here at emphasizing the (in general) different structures of $\mathcal{S}$.
} 
where $\mathcal{S}(\bullet)$ now represents a functional of the form

$$
\mathcal{S}(\bullet)=\left\{\begin{array}{l}
\int_{\mathcal{Z}} P_{t} Q_{z} \mathrm{~d} z \\
\int_{\mathcal{Z}} Q_{t} P_{z} \mathrm{~d} z .
\end{array}\right.
$$

Indeed, invoking Hamilton's principle yields either one of the following sets of Lagrangian equations

$$
\begin{aligned}
\left\{\left(\delta_{Q_{t}} \mathcal{L}\right)_{t}=\right. & \left.\left(L Q_{t}\right)_{t}\right\}-\left\{\delta_{Q} \mathcal{L}=\left(P_{t}\right)_{z}\right\}=0 \\
& \Rightarrow L Q_{t t}=-P_{t z} \\
\left\{\left(\delta_{P_{t}} \mathcal{L}\right)_{t}=\right. & \left.\left(C P_{t}+Q_{z}\right)_{t}\right\}-\left\{\delta_{P} \mathcal{L}=0\right\}=0 \\
& \Rightarrow C P_{t t}=-Q_{z t},
\end{aligned}
$$

or

$$
\begin{aligned}
\left\{\left(\delta_{Q_{t}} \mathcal{L}\right)_{t}=\right. & \left.\left(L Q_{t}+P_{z}\right)_{t}\right\}-\left\{\delta_{Q} \mathcal{L}=0\right\}=0 \\
& \Rightarrow L Q_{t t}=-P_{z t} \\
\left\{\left(\delta_{P_{t}} \mathcal{L}\right)_{t}=\right. & \left.\left(C P_{t}\right)_{t}\right\}-\left\{\delta_{P} \mathcal{L}=\left(Q_{t}\right)_{z}\right\}=0 \\
& \Rightarrow C P_{t t}=-Q_{t z}
\end{aligned}
$$

which after substitution of $Q_{t}=I$ and $P_{t}=V$ both precisely coincide with (1) and (2). For ease of notation, the latter sets of equations can be cast in the general form

$$
\left(\delta_{\boldsymbol{q}_{t}} \mathcal{L}\left(\boldsymbol{q}, \boldsymbol{q}_{t}\right)\right)_{t}-\delta_{\boldsymbol{q}} \mathcal{L}\left(\boldsymbol{q}, \boldsymbol{q}_{t}\right)=\mathbf{0}
$$

where $\boldsymbol{q}=\operatorname{col}(Q, P)$ and $\boldsymbol{q}_{t}=\operatorname{col}\left(Q_{t}, P_{t}\right)$ denote the generalized displacement and generalized velocity coordinates, respectively.

Remark 6 In the light of Remark 4 it is obvious that instead of (41) we could equally well take

$$
\mathcal{S}(\bullet)=-\left\{\begin{array}{l}
\int_{\mathcal{Z}} Q P_{t z} \mathrm{~d} z \\
\int_{\mathcal{Z}} P Q_{t z} \mathrm{~d} z
\end{array}\right.
$$

which can be considered as the distributed-parameter version of (38). A similar discussion applies to (39).

Remark 7 Note that $\mathcal{S}(\bullet)$ can be considered as the infinite-dimensional analogue of a second-degree traditor, which is equivalent to a transformer having as 'turns-ratio' the spatial derivative $\partial / \partial z$. 
In passing on to the Hamiltonian formulation, we now define the conjugate momenta

$$
\boldsymbol{p}=\left(\begin{array}{l}
\Pi \\
\Gamma
\end{array}\right)=\delta_{\boldsymbol{q}_{t}} \mathcal{L}\left(\boldsymbol{q}, \boldsymbol{q}_{t}\right)
$$

and find the Legendre transformation $\mathcal{H}(\boldsymbol{q}, \boldsymbol{p})$ of $\mathcal{L}\left(\boldsymbol{q}, \boldsymbol{q}_{t}\right)$ as

$$
\mathcal{H}(\boldsymbol{q}, \boldsymbol{p})=\int_{\mathcal{Z}} \boldsymbol{p} \cdot \boldsymbol{q}_{t} \mathrm{~d} z-\mathcal{L}\left(\boldsymbol{q}, \boldsymbol{q}_{t}\right)
$$

In the present setting this means that either

$$
\Pi=\left\{\begin{array}{l}
L Q_{t} \\
L Q_{t}+P_{z}
\end{array} \quad \text { and } \Gamma=\left\{\begin{array}{l}
C P_{t}+Q_{z} \\
C P_{t}
\end{array}\right.\right.
$$

results in a Hamiltonian functional

$$
\mathcal{H}(\bullet, \Pi, \Gamma)=\int_{\mathcal{Z}} \overline{\mathcal{H}}(\bullet, \Pi, \Gamma) \mathrm{d} z
$$

with respective Hamiltonian densities

$$
\overline{\mathcal{H}}(\bullet, \Pi, \Gamma)=\left\{\begin{array}{l}
\frac{1}{2 L} \Pi^{2}+\frac{1}{2 C}\left(\Gamma-Q_{z}\right)^{2} \\
\frac{1}{2 L}\left(\Pi-P_{z}\right)^{2}+\frac{1}{2 C} \Gamma^{2}
\end{array}\right.
$$

Furthermore, it is easily checked that the Hamiltonian is conserved since along the trajectories of

$$
\boldsymbol{q}_{t}=\delta_{\boldsymbol{p}} \mathcal{H}(\boldsymbol{q}, \boldsymbol{p}), \quad \boldsymbol{p}_{t}=-\delta_{\boldsymbol{q}} \mathcal{H}(\boldsymbol{q}, \boldsymbol{p})
$$

its time-derivative satisfies $\dot{\mathcal{H}}=0$.

\subsection{A Physical Argument}

As argued in [14], there should be a distinction between a so-called mathematical and a physical Lagrangian. In contrast to a mathematical Lagrangian, a physical Lagrangian for a (non-relativistic) system should not only yield the Euler-Lagrange equations leading to the correct equations of motion, but it should also be expressible as the difference between the kinetic (co-)energy and the potential energy ${ }^{3}$. Furthermore, the associated Hamiltonian should equal the total energy of the system. Some interesting discussions and relaxations concerning these arguments can be found in [9] and [12]. Although the proposed Lagrangian (40), together with (41), yields the correct equations of motion for a lossless transmission line, its form does not equal the difference between the magnetic coenergy and the electric energy (or vice-versa). Apart from the fact that such requirement

\footnotetext{
${ }^{3}$ Or some appropriate generalization, which in case of the transmission line should be the difference between the magnetic (co-)energy and the electric energy, or vice versa.
} 
is reasonable or not, it is important to note that our Hamiltonian (46) does clearly constitute the total (co-)energy in the transmission line since

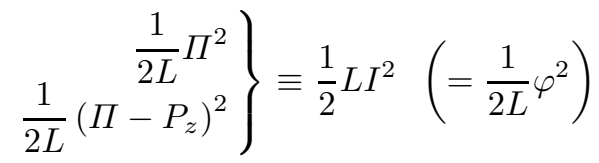

$$
\begin{aligned}
& \left.\frac{\frac{1}{2 C}\left(\Gamma-Q_{z}\right)^{2}}{\frac{1}{2 C} \Gamma^{2}}\right\} \equiv \frac{1}{2} C V^{2}\left(=\frac{1}{2 C} q^{2}\right) \text {. }
\end{aligned}
$$

Note that this result stems from the fact that the coupling Lagrangians $\mathcal{S}$ are nonenergetic and thus vanish in the Legendre transformation from the Lagrangian to the Hamiltonian.

\subsection{Lagrangian Boundary Control System}

In a similar fashion as before, in case of nonzero boundary energy flow we consider the functional (40) as the internal Lagrangian and extend the formulation by adding additional interaction Lagrangians, i.e.,

$$
\mathcal{L}\left[\boldsymbol{q}, \boldsymbol{q}_{t}, \boldsymbol{e}\right]=\mathcal{L}^{\mathrm{int}}\left(\boldsymbol{q}, \boldsymbol{q}_{t}\right)+\mathcal{L}^{0}\left(\bullet, \boldsymbol{e}^{0}\right)+\mathcal{L}^{1}\left(\bullet, \boldsymbol{e}^{1}\right)
$$

and define the following Lagrangian boundary control system:

$$
\left(\delta_{\boldsymbol{q}_{t}} \mathcal{L}\left[\boldsymbol{q}, \boldsymbol{q}_{t}, \boldsymbol{e}\right]\right)_{t}-\delta_{\boldsymbol{q}} \mathcal{L}\left[\boldsymbol{q}, \boldsymbol{q}_{t}, \boldsymbol{e}\right]=\mathbf{0} .
$$

Hence, starting, for example, from $\overline{\mathcal{S}}\left(Q_{z}, P_{t}\right)=P_{t} Q_{z}$, so that the internal Lagrangian reads

$$
\mathcal{L}^{\text {int }}\left(Q, P, Q_{t}, P_{t}\right)=\int_{\mathcal{Z}} \frac{1}{2}\left(L Q_{t}^{2}+C P_{t}^{2}\right) \mathrm{d} z+\int_{\mathcal{Z}} P_{t} Q_{z} \mathrm{~d} z,
$$

and leaving the interaction Lagrangians unprescribed for the moment, yields the Lagrangian boundary control system:

$$
\left(\begin{array}{c}
L Q_{t t} \\
C P_{t t}+Q_{z t} \\
\left(\mathcal{L}_{Q_{t}^{0}}^{0}\right)_{t} \\
\left(\mathcal{L}_{P_{t}^{0}}^{0}\right)_{t} \\
\left(\mathcal{L}_{Q_{t}^{1}}^{1}\right)_{t} \\
\left(\mathcal{L}_{P_{t}^{1}}^{1}\right)_{t}
\end{array}\right)-\left(\begin{array}{c}
-P_{t z} \\
0 \\
\mathcal{L}_{Q^{0}}^{0}-P_{t}^{0} \\
\mathcal{L}_{P^{0}}^{0} \\
\mathcal{L}_{Q^{1}}^{1}+P_{t}^{1} \\
\mathcal{L}_{P^{1}}^{1}
\end{array}\right)=\mathbf{0}
$$

If we now return to the example of a transmission line that is terminated on both ends by independent voltage sources $(\mathrm{VV}) e^{0}=\operatorname{col}\left(E^{0}, 0\right)$ and $e^{1}=\operatorname{col}\left(E^{1}, 0\right)$ it is readily found that in this case $\mathcal{L}^{0}=E^{0} Q^{0}$ and $\mathcal{L}^{1}=-E^{1} Q^{1}$. 
Table 1: Causality configurations of a terminated transmission line for the boundary control system (50), starting from $\overline{\mathcal{S}}=Q_{t} P_{z}$.

\begin{tabular}{c||c|c|c|c||c|c} 
& $e_{1}^{0}$ & $e_{2}^{0}$ & $e_{1}^{1}$ & $e_{2}^{1}$ & $\mathcal{L}^{0}$ & $\mathcal{L}^{1}$ \\
\hline \hline $\mathrm{VV}$ & $E^{0}$ & 0 & $E^{1}$ & 0 & $\left(E^{0}-P_{t}^{0}\right) Q^{0}$ & $-\left(E^{1}-P_{t}^{1}\right) Q^{1}$ \\
$\mathrm{VC}$ & $E^{0}$ & 0 & 0 & $J^{1}$ & $\left(E^{0}-P_{t}^{0}\right) Q^{0}$ & $-J^{1} P^{1}$ \\
$\mathrm{CV}$ & 0 & $J^{0}$ & $E^{1}$ & 0 & $J^{0} P^{0}$ & $-\left(E^{1}-P_{t}^{1}\right) Q^{1}$ \\
$\mathrm{CC}$ & 0 & $J^{0}$ & 0 & $J^{1}$ & $J^{0} P^{0}$ & $-J^{1} P^{1}$
\end{tabular}

Table 2: Causality configurations of a terminated transmission line for the boundary control system (50), starting from $\overline{\mathcal{S}}=P_{t} Q_{z}$.

\begin{tabular}{c||c|c|c|c||c|c} 
& $e_{1}^{0}$ & $e_{2}^{0}$ & $e_{1}^{1}$ & $e_{2}^{1}$ & $\mathcal{L}^{0}$ & $\mathcal{L}^{1}$ \\
\hline \hline $\mathrm{VV}$ & $E^{0}$ & 0 & $E^{1}$ & 0 & $E^{0} Q^{0}$ & $-E^{1} Q^{1}$ \\
$\mathrm{VC}$ & $E^{0}$ & 0 & 0 & $J^{1}$ & $E^{0} Q^{0}$ & $-\left(J^{1}-Q_{t}^{1}\right) P^{1}$ \\
$\mathrm{CV}$ & 0 & $J^{0}$ & $E^{1}$ & 0 & $\left(J^{0}-Q_{t}^{0}\right) P^{0}$ & $-E^{1} Q^{1}$ \\
$\mathrm{CC}$ & 0 & $J^{0}$ & 0 & $J^{1}$ & $\left(J^{0}-Q_{t}^{0}\right) P^{0}$ & $-\left(J^{1}-Q_{t}^{1}\right) P^{1}$
\end{tabular}

On the other hand, if the line is terminated by current sources (CC) we need to select either $\overline{\mathcal{S}}\left(P_{z}, Q_{t}\right)=Q_{t} P_{z}$, or admit for interaction Lagrangians that also depend on the generalized velocities at the boundary, i.e., $\mathcal{L}^{0}=\mathcal{L}^{0}\left(\boldsymbol{q}^{0}, \boldsymbol{q}_{t}^{0}, \boldsymbol{e}^{0}\right)$ and $\mathcal{L}^{1}=\mathcal{L}^{1}\left(\boldsymbol{q}^{1}, \boldsymbol{q}_{t}^{1}, \boldsymbol{e}^{1}\right)$. Indeed, if in the present setting the voltage sources are replaced by current sources $\boldsymbol{e}^{0}=\operatorname{col}\left(0, J^{0}\right)$ and $\boldsymbol{e}^{1}=\operatorname{col}\left(0, J^{1}\right)$, then the interaction Lagrangians need to be set as $\mathcal{L}^{0}=\left(J^{0}-Q_{t}^{0}\right) P^{0}$ and $\mathcal{L}^{1}=-\left(J^{1}-Q_{t}^{1}\right) P^{1}$. This also allows for mixed combinations, i.e., voltage/current (VC) and current/voltage (CV) causalities, providing the four different boundary configurations summarized in Table 1 and 2 . However, as will be demonstrated next, the corresponding Hamiltonian control system formulation does not allow for the mixed cases.

\subsection{Symplectic Hamiltonian Boundary Control System}

As before, one now defines the corresponding Hamiltonian boundary control system starting from the Legendre transformation of the boundary control Lagrangian (49). If the interaction terms $\mathcal{L}^{0}$ and $\mathcal{L}^{1}$ depend only on the generalized displacements then they do not contribute any conjugate boundary momenta to the Legendre transformation. In that case, the internal Hamiltonian is simply taken as (46), with either one of the two densities (47), whereas the interaction Hamiltonians follow verbatim from the respective $\mathcal{L}^{0}$ and $\mathcal{L}^{1}$ defined in Table 1 and 2.

For example, insisting that $\mathcal{L}^{0}=\mathcal{L}^{0}\left(\boldsymbol{q}^{0}, \boldsymbol{e}^{0}\right)$ and $\mathcal{L}^{1}=\mathcal{L}^{0}\left(\boldsymbol{q}^{1}, \boldsymbol{e}^{1}\right)$, the voltage/voltagecontrolled (VV) configuration suggests to select as the internal Hamiltonian the one with 
$\overline{\mathcal{S}}=P_{t} Q_{z}$. This yields a boundary control Hamiltonian of the form

$$
\mathcal{H}\left[Q, \Pi, \Gamma, E^{0}, E^{1}\right]=\int_{\mathcal{Z}} \frac{1}{2 L} \Pi^{2} \mathrm{~d} z+\int_{\mathcal{Z}} \frac{1}{2 C}\left(\Gamma-Q_{z}\right)^{2} \mathrm{~d} z-E^{0} Q^{0}+E^{1} Q^{1},
$$

which has well-defined functional derivatives, as defined in (48), since its variation yields that the natural boundary conditions vanish identically, i.e.,

$$
\begin{aligned}
-E^{0}+\left.\frac{1}{C}\left(\Gamma-Q_{z}\right)\right|_{z=0} & =0 \\
E^{1}-\left.\frac{1}{C}\left(\Gamma-Q_{z}\right)\right|_{z=1} & =0 .
\end{aligned}
$$

Similarly, variation of the total Hamiltonian associated with $\overline{\mathcal{S}}=Q_{t} P_{z}$ given by

$$
\mathcal{H}\left[P, \Pi, \Gamma, J^{0}, J^{1}\right]=\int_{\mathcal{Z}} \frac{1}{2 L}\left(\Pi-P_{z}\right)^{2} \mathrm{~d} z+\int_{\mathcal{Z}} \frac{1}{2 C} \Gamma^{2} \mathrm{~d} z+J^{0} P^{0}-J^{1} P^{1},
$$

yields the natural boundary conditions

$$
\begin{gathered}
J^{0}-\left.\frac{1}{L}\left(\Pi-P_{z}\right)\right|_{z=0}=0 \\
-J^{1}+\left.\frac{1}{L}\left(\Pi-P_{z}\right)\right|_{z=1}=0 .
\end{gathered}
$$

These descriptions can also be merged into a boundary control formulation by introducing a skew-symmetric matrix $[\boldsymbol{F}]=-[\boldsymbol{F}]^{T}$ such that

$$
[\boldsymbol{F}]^{T}\left[\boldsymbol{x}_{t}\right]=\delta_{[\boldsymbol{x}]} \mathcal{H}[\boldsymbol{x}, \boldsymbol{e}],
$$

where $\boldsymbol{x}=\operatorname{col}(\boldsymbol{q}, \boldsymbol{p})$,

$$
[\boldsymbol{F}]=\left(\begin{array}{ccc}
\boldsymbol{F} & & \\
& \boldsymbol{F}^{0} & \\
& & \boldsymbol{F}^{1}
\end{array}\right), \text { with } \boldsymbol{F}=\left(\begin{array}{cc}
\mathbf{0} & \boldsymbol{I}_{2 \times 2} \\
-\boldsymbol{I}_{2 \times 2} & \mathbf{0}
\end{array}\right),
$$

and $\boldsymbol{F}^{0}=\boldsymbol{F}^{1}=\mathbf{0}$.

On the other hand, for a mixed causality like the voltage/current-controlled (VC) configuration, we have for $\overline{\mathcal{S}}=P_{t} Q_{z}$ that $\mathcal{L}^{0}=E^{0} Q^{0}$, but $\mathcal{L}^{1}=-\left(J^{1}-Q_{t}^{1}\right) P^{1}$. As a result, the latter interaction term now contributes a conjugate boundary momentum

$$
[\boldsymbol{p}]=\delta_{\left[\boldsymbol{q}_{t}\right]} \mathcal{L}\left[\boldsymbol{q}, \boldsymbol{q}_{t}, \boldsymbol{e}\right]=\left(\begin{array}{c}
L Q_{t} \\
\frac{C P_{t}+Q_{z}}{0} \\
\frac{0}{P^{1}} \\
0
\end{array}\right)
$$


yielding that the Legendre transformation

$$
\begin{aligned}
\mathcal{H}\left[Q, P, \Pi, \Gamma, E^{0}, J^{1}\right] & =\mathcal{H}^{\mathrm{int}}(Q, \Pi, \Gamma)+P^{1} \frac{\Pi^{1}}{L}-\left\{E^{0} Q^{0}-\left(J^{1}-\frac{\Pi^{1}}{L}\right) P^{1}\right\} \\
& =\mathcal{H}^{\mathrm{int}}(Q, \Pi, \Gamma)-E^{0} Q^{0}+J^{1} P^{1} .
\end{aligned}
$$

Although the latter functional seems a valid Hamiltonian, its functional derivative is only well-defined if the associated boundary terms satisfy a rather unpractical condition. Indeed, since $\Pi^{1}=P^{1}$ we find in terms of the Hamiltonian boundary control system formulation (55) that $\boldsymbol{F}^{0}=\mathbf{0}$, but

$$
\boldsymbol{F}^{1}=\left(\begin{array}{cc|cc}
0 & 0 & -1 & 0 \\
0 & 0 & 0 & 0 \\
\hline 1 & 0 & 0 & 0 \\
0 & 0 & 0 & 0
\end{array}\right) .
$$

This results in the following set of boundary conditions:

$$
\begin{aligned}
-E^{0}+\left.\frac{1}{C}\left(\Gamma-Q_{z}\right)\right|_{z=0} & =0 \\
-\Pi_{t}^{1} & =-\left.\frac{1}{C}\left(\Gamma-Q_{z}\right)\right|_{z=1}\left(=-P_{t}^{1}\right) \\
J^{1} & =0 \\
Q_{t}^{1} & =0 .
\end{aligned}
$$

Clearly, since the boundary conditions need to satisfy $J^{1}=Q_{t}^{1}=0$ there can not be any energy flow through the boundary at $z=1$. For the current/voltage-controlled configuration a similar result can be obtained. A brief explanation for this discrepancy is provided in Section 6. .

\section{On the Role of Dissipation}

So far we have considered only the description of a lossless transmission line. Let us next turn to the case of a lossy line. The transmission equations (1) and (2) then take the form

$$
\begin{aligned}
& L I_{t}+V_{z}=-R I \\
& C V_{t}+I_{z}=-G V
\end{aligned}
$$

where $R$ and $G$ are the distributed resistance and shunt conductance, respectively.

In the context of the Lagrangian formalism, a standard approach is to include dissipative effects by introducing a Rayleigh dissipation function(al). However, in the classical approach, where we have started from an integrated charge description, the corresponding equation of motion only constitutes the lines voltage balance (7). This means that we 
can only include the transversal dissipation effects by considering a Rayleigh dissipation functional of the form

$$
\mathcal{R}\left(Q_{t}\right)=\int_{\mathcal{Z}} \frac{1}{2} R Q_{t}^{2} \mathrm{~d} z
$$

The corresponding equation of motion (7) extends to

$$
L Q_{t t}-\frac{Q_{z z}}{C}=-\delta_{Q_{t}} \mathcal{R}\left(Q_{t}\right)=-R Q_{t}
$$

which in turn coincides with (59).

On the other hand, as also mentioned in Remark 3, invoking a co-Lagrangian description starting from an integrated flux coordinate suggests the introduction of a Rayleigh co-dissipation functional

$$
\mathcal{R}^{*}\left(P_{t}\right)=\int_{\mathcal{Z}} \frac{1}{2} G P_{t}^{2} \mathrm{~d} z .
$$

This yields the current balance (60) in terms of the integrated flux:

$$
C P_{t t}-\frac{P_{z z}}{L}=-\delta_{P_{t}} \mathcal{R}^{*}\left(P_{t}\right)=-G P_{t}
$$

Now, invoking the theory presented in the previous sections, these two separate results can be derived from a single Lagrangian. This means that starting, for example, from an internal Lagrangian of the form (51), we obtain for a lossy line

$$
\begin{aligned}
& L Q_{t t}+P_{t z}=-\delta_{Q_{t}} \mathcal{R}^{\mathrm{int}}\left(Q_{t}, P_{t}\right)=-R Q_{t} \\
& C P_{t t}+Q_{z t}=-\delta_{P_{t}} \mathcal{R}^{\mathrm{int}}\left(Q_{t}, P_{t}\right)=-G P_{t},
\end{aligned}
$$

where $\mathcal{R}^{\mathrm{int}}\left(Q_{t}, P_{t}\right)$ is the total internal Rayleigh dissipation functional defined by

$$
\mathcal{R}^{\text {int }}\left(Q_{t}, P_{t}\right)=\int_{\mathcal{Z}} \frac{1}{2}\left(R Q_{t}^{2}+G P_{t}^{2}\right) \mathrm{d} z .
$$

In a similar fashion as before, it is also possible to define an interaction Rayleigh dissipation functional to include resistances or conductances that occur at the line terminals.

\section{Final Remarks}

In this paper, the classical Lagrangian and Hamiltonian formulation of a uniform transmission line is accommodated to account for nonzero boundary energy flow. The extended classical framework is, however, limited to a line that is terminated on both ends by independent sources of the same type. This restriction stems from the fact that the classical formulation captures only one wave equation for a lossless transmission line in terms of either an integrated charge or flux density and has motivated the search for alternative Lagrangian variational principles that yield both the transmission line equations simultaneously. The approach is inspired by an infinite-dimensional generalization of a network-theoretic concept called the traditor. 
Although the new Lagrangian formulation allows for mixed boundary conditions, the associated Hamiltonian formulation still only allows both ends to be terminated either by independent voltage sources or current sources, but not both. This discrepancy can be explained as follows. Starting, for example from Table 2, the necessary interaction Lagrangians for a voltage/current (VC) causality are $\mathcal{L}^{0}=E^{0} Q^{0}$ and $\mathcal{L}^{1}=-\left(J^{1}-Q_{t}^{1}\right) P^{1}$. The term $Q_{t}^{1} P^{1}$ can be considered as a boundary version of an $\mathcal{S}$-type function and serves as a 'correction' to moderate the configuration for the inclusion of a current source. Since $\mathcal{S}$-type functions are non-energic in nature (cf. Subsection 4.2.) they vanish in the transition from the Lagrangian to the energic Hamiltonian framework. Indeed, the Legendre transformation of $\mathcal{S}^{1}=Q_{t}^{1} P^{1}$ yields

$$
Q_{t}^{1} \mathcal{S}_{Q_{t}^{1}}^{1}-\mathcal{S}^{1}=0 .
$$

One possibility to adapt the Hamiltonian framework for the inclusion of mixed boundary sources is by adding appropriate boundary terms to the original energic Hamiltonian and construct a generalized Hamiltonian including non-energic contributions. This invokes the need of Lagrange multipliers and leads to an implicit Hamiltonian description. A similar idea in the lumped-parameter case is used in [18].

Another limitation of the classical framework is the inclusion of dissipation if both resistance and shunt conductance are considered. As shown in [12], an alternative way to account for losses is to consider a modified version of Hamilton's principle using a weighted Lagrangian density with a time-dependent exponential factor. However, apart from the fact that one runs into problems when dealing with nonlinear resistances and/or shunt conductances, the associated Hamiltonian does not have the interpretation of the total stored energy in the system. Since the present formulation provides both transmission line equations simultaneously, energy dissipation can be included in a clear and transparent manner introducing the usual Rayleigh dissipation functional.

\section{REFERENCES}

[1] R. Abraham, J.E. Marsden, and T. Ratiu. Manifolds, Tensor Analysis, and Applications, Volume 75. Springer-Verlag, New York, second edition, 1988.

[2] G.D. Birkhoff. Dynamical Systems. Am. Math. Soc. (AMS), 1927.

[3] R.K. Brayton and W.L. Miranker. A stability theory for nonlinear mixed-initial boundary value problems. Arch. Ratl. Mech. and Anal., 17(5):358-376, December 1964.

[4] R.W. Brockett. Control theory and analytical mechanics. in Geometric Control Theory (eds. C. Martin, R. Hermann), Vol. VII of Lie Groups: History, Frontiers and Applications, Math. Sci. Press., pages 1-46, 1977.

[5] E.C. Cherry. Some general theorems for nonlinear systems possessing reactance. Phil. Mag., Ser. 7, 42(333):1161-1177, October 1951.

[6] R. Courant and D. Hilbert. Methods of Mathematical Physics, Vol. I. Interscience Publishers, Inc., New York, 1953.

[7] S. Duinker. Traditors, a new class of non-energetic non-linear network elements. Philips Res. Repts, 14:29-51, 1959. 
[8] H. Goldstein. Classical Mechanics. Addison-Wesley, Reading, MA, 1980.

[9] D.H. Kobe and G. Reali. Lagrangians for dissipative systems. Am. J. Phys., 54(11):997999, November 1986.

[10] A.G.J. MacFarlane. Dynamical System Models. George G. Harrap \& Co. Ltd., 1970.

[11] L.M. Magid. Electromagnetic Fields, Energy and Waves. New York, Wiley, 1972.

[12] M.J. Morgan. Lagrangian formulation of a transmission line. Am. J. Phys., 56(7):639-643, July 1988.

[13] H. Nijmeijer and A.J. Van der Schaft. Nonlinear Dynamical Control Systems. SpringerVerlag New York Inc., 1990.

[14] J.R. Ray. Lagrangians and systems they describe: how not to treat dissipation in quantum mechanics. Am. J. Phys., 47(7):626-629, 1979.

[15] A.J. Van der Schaft and B.M. Maschke. Hamiltonian formulation of distributed-parameter systems with boundary energy flow. Journal of Geometry and Physics, 42:166-194, 2002.

[16] L. Weiss and W. Mathis. A Hamiltonian formulation for complete nonlinear RLC-networks. IEEE Trans. Circuits and Systems - I: Fund. Theory and Appl., 44(9):843-846, September 1997.

[17] H. Yoshimura and J. E. Marsden. Dirac structures and implicit Lagrangian systems in electric networks. Proceedings of the 17th International Symposium on Mathematical Theory of Networks and Systems (Kyoto, Japan), pp. 1444-1449, 2006.

[18] H. Yoshimura, and J. E. Marsden. Dirac structures and the Legendre transformation for implicit Lagrangian and Hamiltonian systems. Lagrangian and Hamiltonian Methods in Nonlinear Control 2006, volume 366 of Lecture Notes in Control and Information Science Series, pp. 233-247, Springer-Verlag, 2007. 